Dig Dis 1995;13:389

\title{
Vibrio vulnificus Skin Lesion
} S.

Department of Infectious Diseases, Division of Internal Medicine, Mayo Clinic, Jacksonville, Fla.

Salvador Alvarez-Elcoro, MD, Associate Professor of Medicine, Department of Infectious Diseases, Division of Internal Medicine, Mayo Clinic, 4500 San Pablo Road, Jacksonville, FL 32224 (USA)

An elderly male with end-stage liver cirrhosis and a recent history of raw oyster ingestion presented with fever and skin lesions. The aspirate from the skin lesions and the blood cultures grew Vibrio vulnificus. In spite of intensive supportive therapy and intravenous antibiotics, the patient expired. In patients with cirrhosis, infection with V. vulnificus results in hema-togenous seeding of the skin, usually of the lower extremities. Cutaneous lesions begin with edema and erythema, followed by vesicles and bullae formation and ultimately necrosis and sloughing.

(C)1995

S. Karger AG, Basel

0257-2753/95/

0136-0389S8.00/0 